Rot, gelb, grün - Patienten bewerten ihren Blutdruck selbst

Djalali, S ; Chmiel, C

Posted at the Zurich Open Repository and Archive, University of Zurich ZORA URL: https://doi.org/10.5167/uzh-116940

Journal Article

Published Version

Originally published at:

Djalali, S; Chmiel, C (2015). Rot, gelb, grün - Patienten bewerten ihren Blutdruck selbst. PrimaryCare:392393. 


\title{
Rot, gelb, grün - Patienten bewerten ihren Blutdruck selbst
}

\section{Sima Djalali, Corinne Chmiel}

\author{
In PrimaryCare haben wir 2013 über den Auftakt der CoCo-Studie berichtet [1], in der \\ ein neuer Ansatz im Management von Hypertoniepatienten getestet werden sollte. \\ Jetzt liegen die Ergebnisse vor [2].
}

CoCo steht für «Colour-coded Blood Pressure Control». In dieser Interventionsstudie wurde erprobt, wie es sich auswirkt, wenn Hypertoniepatienten ein farbcodiertes Blutdrucktagebuch zur Verfügung gestellt bekommen, das ihnen dabei hilft, Heimblutdruckmessungen nicht nur zu dokumentieren, sondern auch selbständig zu interpretieren.

\section{Kompetente Patienten sind adhärenter}

Die Hypothese dahinter ist, dass Patienten, die kompetent im Umgang mit ihrer Krankheit sind, aktiver in ihre Therapieplanung involviert sind, die Therapieanweisungen stärker befolgen und daher eine bessere Krankheitskontrolle erzielen. Den Verlauf klinischer Schlüsselparameter ihrer Erkrankung - in diesem Fall Blutdruckwerte - selbständig monitorieren zu können, stärkt die Kompetenz der Patienten.

\section{Blutdruck selber messen}

Dazu führten wir zwischen Oktober 2009 und November 2011 eine randomisiert kontrollierte Studie in den Regionen Zürich und St.Gallen durch. 30 Hausärzte unterstützten das Projekt und schlossen insgesamt 137 Patienten im Alter über 18 Jahre mit einem Blutdruck von $>140 \mathrm{~mm} \mathrm{Hg}$ systolisch und/oder $>90 \mathrm{~mm}$ Hg diastolisch ein. Alle Patienten erhielten ein Heimblutdruckmessgerät und ein Blutdrucktagebuch. Patienten der Kontrollgruppe erhielten zudem ein Schwarzweiss-Blutdrucktagebuch mit einer simplen Tabelle zum Notieren der systolischen und diastolischen Blutdruckwerte sowie der Pulsfrequenz.

\section{Blutdruck selber einschätzen}

Die Probanden der Interventionsgruppe erhielten dagegen ein Blutdrucktagebuch, in dem die vorgedruckte Tabelle nach einem Ampelschema farbig eingeteilt war. Für Messwerte bis maximal 140/90 mm Hg war in der Tabelle eine grüngefärbte Kolonne mit der Überschrift «Normal» vorgesehen. Für Messwerte zwischen 140-180 und 90-110 mm Hg war dagegen eine gelb hinterlegte Kolonne mit der Überschrift "Achtung» bestimmt, und Messwerte $>180 />110 \mathrm{~mm} \mathrm{Hg}$ sollten in eine rot hinterlegte Kolonne mit der Überschrift «Gefahr» eingetragen werden (Abb. 1). Die Blutdrucktagebücher sollten die Patienten in den nächsten sechs

\begin{tabular}{|c|c|c|c|c|c|c|c|c|}
\hline \multirow[t]{2}{*}{ Datum } & \multirow[t]{2}{*}{ Zeit } & \multicolumn{2}{|c|}{ Normal } & \multicolumn{2}{|c|}{ Achtung } & \multicolumn{2}{|c|}{ Gefahr } & Puls \\
\hline & & $\begin{array}{l}\text { Oberer } \\
\text { Wert } \\
\text { bis } 140\end{array}$ & $\begin{array}{l}\text { Unterer } \\
\text { Wert } \\
\text { bis } 90\end{array}$ & $\begin{array}{l}\text { Oberer } \\
\text { Wert } \\
140-180\end{array}$ & $\begin{array}{l}\text { Unterer } \\
\text { Wert } \\
90-110\end{array}$ & $\begin{array}{l}\text { Oberer } \\
\text { Wert } \\
\text { über } 180\end{array}$ & $\begin{array}{l}\text { Unterer } \\
\text { Wert } \\
\text { über } 110\end{array}$ & \\
\hline & & & & & & & & \\
\hline & & & & & & & & \\
\hline & & & & & & & & \\
\hline & & & & & & & & \\
\hline
\end{tabular}

Abbildung 1: Der CoCo-Blutdruckpass ermöglicht es dem Patienten, seinen Blutdruck mit einem Blick selbst einzuschätzen. 
Monaten ausfüllen und dafür mindestens einmal am Morgen vor der Medikamenteneinnahme nach mindestens fünf Minuten Ruhe im Sitzen den Blutdruck messen. Um Messfehler zu minimieren, wurden alle Patienten im korrekten Umgang mit dem Gerät geschult. Zu Beginn, nach drei und nach sechs Monaten wurde der Blutdruck wiederum durch den Hausarzt gemessen und gegebenenfalls die Therapie angepasst.

\section{Effekt in beiden Gruppen}

Wie sich zeigte, war der Blutdruck innerhalb der beiden Studiengruppen nach sechs Monaten signifikant gesunken; in der Interventionsgruppe im Schnitt um $16 \mathrm{~mm} \mathrm{Hg}$ systolisch und $8 \mathrm{~mm} \mathrm{Hg}$ diastolisch, in der Kontrollgruppe um $13 \mathrm{~mm} \mathrm{Hg}$ systolisch und $9 \mathrm{~mm} \mathrm{Hg}$ diastolisch. Der Unterschied zwischen den beiden Gruppen war jedoch nicht signifikant. Zum Teil lässt sich dies dadurch erklären, dass Patienten beider Gruppen durch die Studienteilnahme eine Intervention im Sinne einer für sie neuen Monitoring-Situation mit geplanten Kontrollterminen beim Hausarzt erfuhren. Dies allein kann ihre Motivation zum aktiven Blutdruckmanagement gesteigert haben - unabhängig vom Effekt des Farbschemas. So wurde auch in beiden Gruppen eine sehr starke Adhärenz beobachtet: Die Patienten füllten das Blutdrucktagebuch jeweils im Schnitt an mehr als 96\% aller Studientage aus.

\section{Ampelschema hat doch Vorteile}

Man kann also den Schluss ziehen, dass das Konzept der Heimblutdruckmessung allein bereits erfolgreich ist. Was sich allerdings zwischen Interventions- und Kontrollgruppe signifikant unterschied, war die Anzahl von Patienten, die eine optimale Blutdruckkontrolle $<140 /<90 \mathrm{~mm}$ Hg erzielten. In der Interventionsgruppe erreichten $42 \%$ dieses Ziel, in der Kontrollgruppe lediglich $25 \%$ der Teilnehmer. Auch zeigte sich in der Analyse der Heimmesswerte über die Zeit, dass die Senkung des systolischen Blutdrucks mit der Verwendung eines farbcodierten Blutdrucktagebuchs assoziiert war. Möglicherweise braucht es länger als sechs Monate Beobachtungszeit oder eine grössere Studienpopulation, damit das Konzept des Ampelschemas einen statistisch signifikanten Zusatznutzen gegenüber einem normalen Blutdrucktagebuch zeigt.

\section{Studie fertig, aber Hausärzte machen weiter}

Interessant wäre auch zu untersuchen, wie es sich auswirkt, wenn die Patienten nicht nur das farbcodierte Blutdrucktagebuch nutzen, sondern auch befähigt werden, ihre Medikamentendosen entsprechend selbst zu titrieren. Dies wird bei Asthmatikern zum Beispiel heute schon erfolgreich durchgeführt. Es gibt zwar Studien bei Blutdruckpatienten, die die Effektivität der Selbsttitration untersucht haben, jedoch lediglich in Verbindung mit normalen Blutdrucktagebüchern.

Für viele der teilnehmenden Hausärzte steht nach Abschluss der CoCo-Studie jedenfalls fest, dass sie die farbcodierten Tagebücher weiterhin an ihre Patienten abgeben werden, da sie und ihre Patienten so gute Erfahrungen damit gemacht haben. Entsprechende Kopiervorlagen stehen jetzt nach Studienende natürlich allen interessierten Kolleginnen und Kollegen zur Verfügung und können beim Institut für Hausarztmedizin per E-Mail angefordert werden.

\section{Literatur}

1 Djalali S, Chmiel C. Viel hilft viel? Nicht bei ungenügend kontrollierter Hypertonie. PrimaryCare. 2013;13(20):367.

2 Chmiel C, Senn O, Rosemann T, Del Prete V, Steurer-Stey C. CoCo trial: Color-coded blood pressure Control, a randomized controlled study. Patient Preference and Adherence. 2014;8:1-10.

\section{PrimaryResearch - das Fenster zur Forschung}

In einer Artikelserie stellen wir die Forschungsarbeiten vor die das Institut für Hausarztmedizin der Universität Zürich (IH$A M Z$ ) seit seiner Gründung publiziert hat. Die Originalarbeiten sind entweder open access zugänglich oder beim jeweiligen Autor, der die Studien hier vorstellen wird, auf Anfrage erhältlich.

Die Ergebnisse geben einen spannenden Einblick in die täglichen Herausforderungen, aber auch die Leistung der Hausarztmedizin.

An dieser Stelle ein herzlicher Dank an alle Kolleginnen und Kollegen, die sich an den jeweiligen Projekten beteiligen und die hier präsentierten Ergebnisse erst ermöglicht haben!
Universität Zürich $^{\text {vin }}$

Institut für Hausarztmedizin 